\title{
Insufficient sensitivity of RNA dependent RNA polymerase Gene of SARS-CoV-2 viral genome as Confirmatory Test using Korean COVID-19 cases
}

\author{
Jaegyun Lim $^{2+}$, Choon-Mee Kim ${ }^{1+}$, KyungHee Lee ${ }^{7+}$, Jun-Won Seo ${ }^{1}$, Na-Ra Yun ${ }^{1}$, You Mi Lee ${ }^{1}$, Wang Jun \\ Lee $^{4}$, Moon Jung Kim ${ }^{5}$, Yu Min Kang ${ }^{6}$, Young Gyu Kang ${ }^{9}$, Dong-ki Lee ${ }^{9}$, Baeckseung Lee ${ }^{3 *}$, Soyoun Kim ${ }^{7,8 *}$, \\ Dong-Min $\mathrm{Kim}^{1 *}$ \\ ${ }^{1}$ College of Medicine, Chosun University, Gwangju, Republic of Korea; \\ ${ }^{2}$ Department of Laboratory Medicine, Myongji Hospital, Hanyang University College of Medicine, ${ }^{3}$ New Horizon Cancer \\ Institute, ${ }^{4}$ Myongji Hospital, Department of General Surgery, Myongji Hospital, ${ }^{5}$ Department of Laboratory Medicine, \\ Myongji Hospital, Hanyang University College of Medicine, ${ }^{6}$ Department of Infectious Diseases, Myongji Hospital, Goyang, \\ Republic of Korea; \\ ${ }^{7}$ PCL Inc., Seoul, Republic of Korea; \\ ${ }^{8}$ Department of Biomedical Engineering, Dongguk University, Seoul, Republic of Korea; \\ ${ }^{9}$ Department of Chemistry, Sungkyunkwan University, Suwon, Republic of Korea
}

+Equally contributed to this work.

*Co-Correspondence:

Dong-Min Kim, M.D.

College of Medicine, Chosun University, 588 Seosuk-dong, Dong-gu, Gwangju 61453, Republic of Korea. Tel:. 82-62-220-3108; Fax: 82-62- 234-965; E-mail address: drongkim@ @chosun.ac.kr Soyoun Kim, Ph.D.

Founder, CEO of PCL Inc., 17 $7^{\text {th }}$ Floor, 128 Beobwon-Ro, Songpa-Gu, Seoul, 05854 Korea. E-mail address: $\underline{\text { skim@pclchip.com }}$

Baeckseung Lee, Ph.D.

Guest Researcher, New Horizon Cancer Institute, Myongji Hospital, 55, Hwasu-ro 14beon-gil,

Deogyang-gu, Goyang-si, Gyeonggi-do, Republic of Korea. E-mail address: baeckseung@gmail.com

Keywords: SARS-CoV-2, Korean COVID-19, Wuhan Corona virus, real time PCR Ct Value, Sensitivity, False Negative 


\section{Insufficient sensitivity of RNA dependent RNA polymerase Gene of SARS-CoV-2 viral genome as Confirmatory Test using Korean COVID-19 cases}

Since mid-December of 2019, coronavirus disease 2019 (COVID-19) has been spreading from Wuhan, China. As of February 21, total 75,773 confirmed cases worldwide have spread to more than two dozen countries.

The first confirmed COVID-19 patients in South Korea are those who came from or visited China. Lately, secondary and tertiary spreads have occurred, and the speed of transmission is accelerating. South Korea has 104 confirmed cases of the COVID-19 and first death case broke. Investigations are underway worldwide to better understand transmission dynamics.

Transmission of COVID-19 can occur early in the course of infection since SARS-CoV-2 viral loads in asymptomatic patients are similar to that in the symptomatic patients. ${ }^{1}$ This indicates asymptomatic or minimally symptomatic patients with low levels of detectable viral RNA can transmit the COVID-19. ${ }^{2}$ Therefore, more sensitive diagnostic methods are needed to detect early phase of the infection to prevent secondary or tertiary spreads.

Currently, the detection method for SARS-CoV-2 is based on viral RNA detection using Reverse Transcribed Real-Time PCR (RT-PCR). WHO initially distributed the guideline for confirming COVID-19, based on RNA dependent RNA polymerase (RdRP) viral RNA gene detecting RT-PCR method. ${ }^{3}$ Accordingly, Korea Centers for Disease Control \& Prevention (KCDC) has used confirmatory RT-PCR based on RdRP gene. On the other hand, US CDC recommended to use SARS-CoV-2 nucleocapsid protein (N) genes instead of RdRP gene as a confirmatory test. ${ }^{4}$

Here, we compare the RT-PCR confirmatory test results using two different SARS-CoV-2 viral RNAs from two Korean COVID-19 confirmed cases. Figure 1A shows that RT-PCR targeting the $\mathrm{N}$ gene is more sensitive than targeting the RdRP gene by 7- 43 fold, based on $\mathrm{Ct}$ value difference, in SARS-CoV-2 whole-genome RNA of Korean COVID-19 cases. Moreover, RdRP gene-based confirmatory test could not detect the virus from case \# 17 which led to false negatives in the early stage.

According to this information, we further formulated the $\mathrm{N}$ genes $(\mathrm{N} 1+\mathrm{N} 3)$ with highest sensitivity for RT-PCR confirmatory test and follow sensitivity of one specimen from Korean COVID case \#3. ${ }^{5}$ In the case of \#3, during administration of anti-HIV agent, 
lopinavir/ritonavir, RdRP gene confirmatory test (KogeneBiotech, Korea) was negative. However, confirmatory test using the $\mathrm{N}$ gene (PCL Inc.) turned out to be positive in Day 10 (Fig 1B). This implicates that low viral titer cannot be detected from RdRP gene confirmatory test currently used in Korea according to WHO guideline.

Protein-coding RNAs in cells differ in their absolute quantity and stability. In this regard, in the RT-PCR-based diagnostic method, it is desirable to detect the viral target RNA with high abundance and stability to maximize detection sensitivity and accuracy. Our results demonstrate that in the case of SARS-CoV-2, RT-PCR targeting $\mathrm{N}$ gene show lower $\mathrm{Ct}$ value and more reproducible detection than RdRP, which might reflect the different RNA abundance and/or stability. Further study is needed to confirm this hypothesis.

In conclusion, to prevent the further spread of COVID-19 by the early-stage patient, WHO should consider recommending the use of $\mathrm{N}$ gene rather than RdRP gene for confirmatory test.

Figure 1. RT-PCR assay targeting viral $\mathrm{N}$ gene can increase the sensitivity of detection of COVID-19

A. RT-PCR assay targeting the RdRP gene (WHO, KCDC, KogeneBiotech) is less sensitive than the one targeting $\mathrm{N}$ genes (CDC, PCL Inc). In the case of Whole Genome (SARS-CoV-2 RNA), N gene can detect the virus with sensitivity increased by $\sim 3$ fold (Ct Value difference 14) compared with the RdRP gene. In the case of Korean COVID-19 Case \#17, N gene can detect the virus with $\sim 10$ fold increased sensitivity (Ct Value difference 3.4) compared with the RdRP gene. In the case of Korean COVID-19 Case \#22, the sensitivity difference is $\sim 7$ fold (Ct Value difference 2.3).

B. RT-PCR assay targeting a mixture of $\mathrm{N}$ genes(N1+N3) show the highest sensitivity (PCLMD ${ }^{\mathrm{TM}}$ nCoV one step RT-PCR Kit from PCL Inc, Seoul, Korea) and confirms Korean COVID-19 case \#3, who was diagnosed as false-negative by the RdRP test. Day 10 after the symptom emerged, viral RNA from COVID-19 Case \#3 patient can be detected by N gene-based RT-PCR, but not by RdRP gene-based RTPCR. 


\section{References}

1. Rothe C, Schunk M, Sothmann P, et al. Transmission of 2019-nCoV Infection from an Asymptomatic Contact in Germany. N Engl J Med 2020.

2. Zou L, Ruan F, Huang M, et al. SARS-CoV-2 Viral Load in Upper Respiratory Specimens of Infected Patients. N Engl J Med 2020.

3. Centers for Disease Control and Prevention, Respiratory Viruses Branch, Division of Viral Diseases. 2019-novel coronavirus (2019-nCoV) real-time RT-PCR panel primers and probes. 2020 (https://www.cdc.gov/coronavirus/2019-ncov/downloads/rt-pcr-panel-primerprobes.pdf).

4. Centers for Disease Control and Prevention, Respiratory Viruses Branch, Division of Viral Diseases. Real-time RT-PCR panel for detection 2019-novel coronavirus. 2020 (https://www.cdc.gov/coronavirus/2019-ncov/downloads/rt-pcr-panel-for-detectioninstructions.pdf)

5. Lim J, Jeon S, Shin HY, et al. Case of the Index Patient Who Caused Tertiary

Transmission of COVID-19 Infection in Korea: the Application of Lopinavir/Ritonavir for the Treatment of COVID-19 Infected Pneumonia Monitored by Quantitative RT-PCR. J Korean Med Sci 2020;35:e79.

\section{Ethics Statement}

Myongji Hospital Institutional Review Board (IRB) approved this study (No. IRB 2020-01027 ) and written informed consent was given by the patient.

\section{Author Disclosure Statement}

The authors have nothing to disclose. 